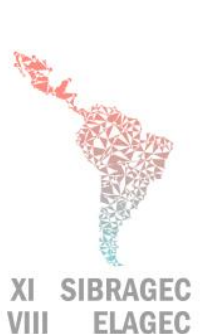

VIII ELAGEC
XI SIMPÓSIO BRASILEIRO DE GESTÃO E ECONOMIA DA CONSTRUÇÃO

VIII ENCUENTRO LATINOAMERICANO DE GESTIÓN

Y ECONOMÍA DE LA CONSTRUCCIÓN

Do conhecimento à ação: práticas avançadas de gestão da produção

Londrina, Paraná, Brasil. 23 a 25 de Outubro de 2019

\title{
AVALIAÇÃO DO ESTADO DE IMPLEMENTAÇÃO DA CONSTRUÇÃO ENXUTA EM UMA OBRA BRASILEIRA
}

\author{
RODEGHERI (1), Priscila Mirapalhete; SERRA (2), Sheyla Mara Baptista; \\ LORENZON (3), Itamar Aparecido
}

(1) Programa de Pós-Graduação em Engenharia Civil. Universidade Federal de São Carlos (UFSCar), (21) 98296 3259, priscilamr@ufscar.br (2) Departamento de Engenharia Civil, UFSCar, sheylabs@ufscar.br, (3) Departamento de Engenharia Civil, UFSCar, itamar@ufscar.br

\begin{abstract}
Lean Construction (LC) is an important management philosophy and its efficiency should be measured as well as another management strategy. Many Measurement Models (MM) can be found to indicate company maturity in LC, however just few of them is suitable for each scenario. In this paper, a case study is carried out applying one international MM in one Brazilian project to verify its suitability. The selected MM were developed by American Community of Practice of Lean Construction Institute (LCI), called LCI Lean IPD Health and Maturity Assessment Tool. This MM were considered proper to this project context and providing a guideline to improve $L C$.
\end{abstract}

Keywords: Lean Construction Institute, Assessment Tool, Integrated Project Delivery, Lean IPD Health and Maturity Assessment Tool.

\section{INTRODUÇÃO E REFERENCIAL TEÓRICO}

O cenário econômico brasileiro exige que as empresas sejam eficientes e competitivas em todas áreas, inclusive na Indústria da Construção Civil (ICC). Faz-se fundamental investimentos em tecnologia e modelos de gestão que propõe mais eficiência e reduzem o desperdício (BÖES et al., 2018). A Construção Enxuta (CE) destaca-se por apresentar mudanças que aprimoram as áreas social, econômica e do ambiente empresarial (GONTIJO; SANTANA; PRADO, 2018) e traz vantagens ao longo do processo de evolução dessa filosofia na empresa (SOUSA; CÂNDIDO; BARROS NETO, 2017).

Para Zhou (2016), a CE vincula-se ao gerenciamento, fornecendo crescimento e vantagens competitivas por meio da redução de custos, estoques, ativos, otimizando processos internos e procedimentos em diversas áreas. Gomes, Cavalcante Filho e Barros Neto (2018) relacionam a CE com premissas de gestão de negócios, como industrialização, modulação, previsão de demanda e sustentabilidade.

A gestão empresarial precisa ser suportada por sistemas, métodos e ferramentas para efetiva implementação, desenvolvimento e avaliação, segundo Lorenzon (2008), diversos fatores mostram que se deve aferir não só os indicadores financeiros, mas também os não-financeiros. Modelos Mensuradores (MM) cumprem esse papel e podem avaliar uma gama de fatores como processos internos, satisfação do cliente,

RODEGHERI, P.M.; SERRA, S.M.B.; LORENZON, I.A. Avaliação do estado de implementação da construção enxuta em uma obra brasileira. In: SIMPÓSIO BRASILEIRO DE GESTÃO E ECONOMIA DA CONSTRUÇÃO, 11., 2019, Londrina. Anais [...]. Porto Alegre: ANTAC, 2019. Disponível em: https://www.antaceventos.net.br/index.php/sibragec/sibragec2019/paper/view/396 


\section{SIBRAGEC - ELAGEC 2019 - de 23 a 25 de Outubro - LONDRINA - PR}

riscos, aprendizado e gestão de mudanças, além do aspecto financeiro. Yu et al. (2007) consideram fundamental escolher um MM adequado ao cenário em análise, para isso, é importante a aplicação de $\mathrm{MM}$ em diferentes projetos para a validação de sua abrangência em cenários distintos.

Francisco, Serra e Lorenzon (2012) pontuam que a preocupação com desperdícios e custos aumentou e, com isso, a adaptação do Pensamento Enxuto (PE) para a construção civil apoiou a melhoria da produtividade e qualidade dos empreendimentos. A disseminação do PE ocorreu, principalmente, após as publicações de Koskela (1992) e Womack e Jones (1997) que tornaram necessário o desenvolvimento de MM focados à implantação da CE. Os MM avaliam a aderência da empresa aos princípios enxutos de forma pontual ou generalizada, verificando o estado atual e progresso ao longo da jornada (SOUSA; CÂNDIDO; BARROS NETO, 2017).

Internacionalmente, Sainth, Varghese e Raghavan (2018) apresentaram avaliação da aplicação e adaptação da CE na Índia e constataram baixo nível de conhecimento e aplicação desta filosofia pelas construtoras. Salvatierra et al. (2015) apresentam dados de avançada aplicação da CE no Chile e estimam que $90 \%$ dos profissionais que trabalham com a filosofia consideram ela fundamental para o crescimento da empresa. Outra experiência positiva apresenta o sucesso na exigência e apoio a aplicação da CE em fornecedores de pequeno-médio porte da empresa pública de rodovias da Inglaterra (TEZEL; KOSKELA; AZIZ, 2017). Isso mostra como a aplicação da CE possui graus distintos em diferentes locais.

Sendo assim, constata-se a efetiva importância da validação de MM que avaliem o desenvolvimento da aplicação das práticas do PE de forma adequada a cada cenário analisado. Portanto, o objetivo desse artigo é verificar a aptidão da ferramenta de avaliação desenvolvida pela Comunidade de Práticas do Instituto Americano de Construção Enxuta (Lean Construction Institute - LCI) chamada Ferramenta de avaliação da maturidade Lean IPD (originalmente LCI Lean Integrated Project Delivery (IPD) Health and Maturity Assessment Tool, em inglês) (LEAN CONSTRUCTION INSTITUTE, 2016), em um empreendimento brasileiro desenvolvido por uma empresa de pequeno-médio porte, apresentando a adaptabilidade do MM ao projeto avaliado e ao uso das práticas de CE nesse empreendimento.

\section{MÉTODO DE ESTUDO}

A ferramenta de avaliação da Maturidade Lean IPD do LCI avalia as melhores práticas de $\mathrm{CE}$ podendo ser aplicada a projetos específicos, equipes ou de forma corporativa. $\mathrm{O}$ modelo considera que a força do grupo está nos indivíduos (LEAN CONSTRUCTION INSTITUTE, 2016) e apresenta uma ferramenta auxiliar de avaliação pessoal e individual que fornece apoio ao modelo principal, porém esta não pode ser aplicada aos atores do empreendimento em questão.

A Figura 1 apresenta resumidamente o processo de desenvolvimento deste artigo. Inicialmente foi realizada uma revisão bibliográfica em base de dados científicos assim como em comitês e instituições vinculadas a CE buscando por MM amplamente difundidos. Diversas ferramentas foram localizadas e a desenvolvida pelo LCI americano foi escolhida para este estudo por avaliar grande amplitude de práticas enxutas e direcionar o caminho a seguir na jornada da CE. Após isso foi definido que o estudo de caso seria realizado em uma obra e não na empresa como um todo. $O$ empreendimento avaliado em questão encontra-se localizado no interior do estado de 
São Paulo e consiste em um condomínio residencial constituído por cinquenta casas. $\mathrm{O}$ empreendimento foi concebido por investidores e recebe fomento de programa federal para construção de moradias populares. Na sequência, o MM escolhido foi aplicado ao projeto em Maio de 2018 e, com os resultados obtidos, foi possível verificar a compatibilidade do modelo ao grau de desenvolvimento da CE nesse empreendimento.

\section{Figura 1 - Desenvolvimento do artigo}

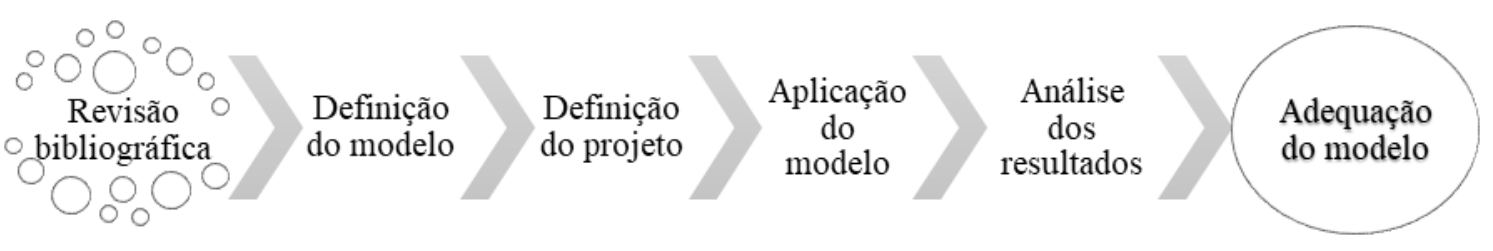

Fonte: Elaboração própria.

O modelo utilizado consiste em um arquivo de planilhas eletrônicas de aplicação intuitiva disponível no site do LCI, e avalia três pontos principais: Transformação; Entrega Integrada de Projeto (Integrated Project Delivery - IPD) e Entrega de projeto enxuta. O último item é subdividido em avaliação do Gerenciamento e métodos e avaliação do Sistema de Planejamento Último (Last Planner System - LPS), conforme apresentado na Figura 2. 191 atributos são avaliados em uma escala de 0 a 5 pontos e distribuídos em 36 características que compõe os pontos principais. Quatro gráficos radar são utilizados para apresentar os resultados, sendo três para cada ponto principal mostrando as características e outro gráfico radar mostra a visão geral do modelo com os quatro pontos iniciais. A escala de seis pontos de avaliação é distribuída homogeneamente e considera os seguintes níveis de conhecimento e aplicação da filosofia: disperso, atento, aprendendo, competente, ensinando, inovando (LEAN CONSTRUCTION INSTITUTE, 2016).

Figura 2 - Ferramenta de avaliação da maturidade Lean IPD

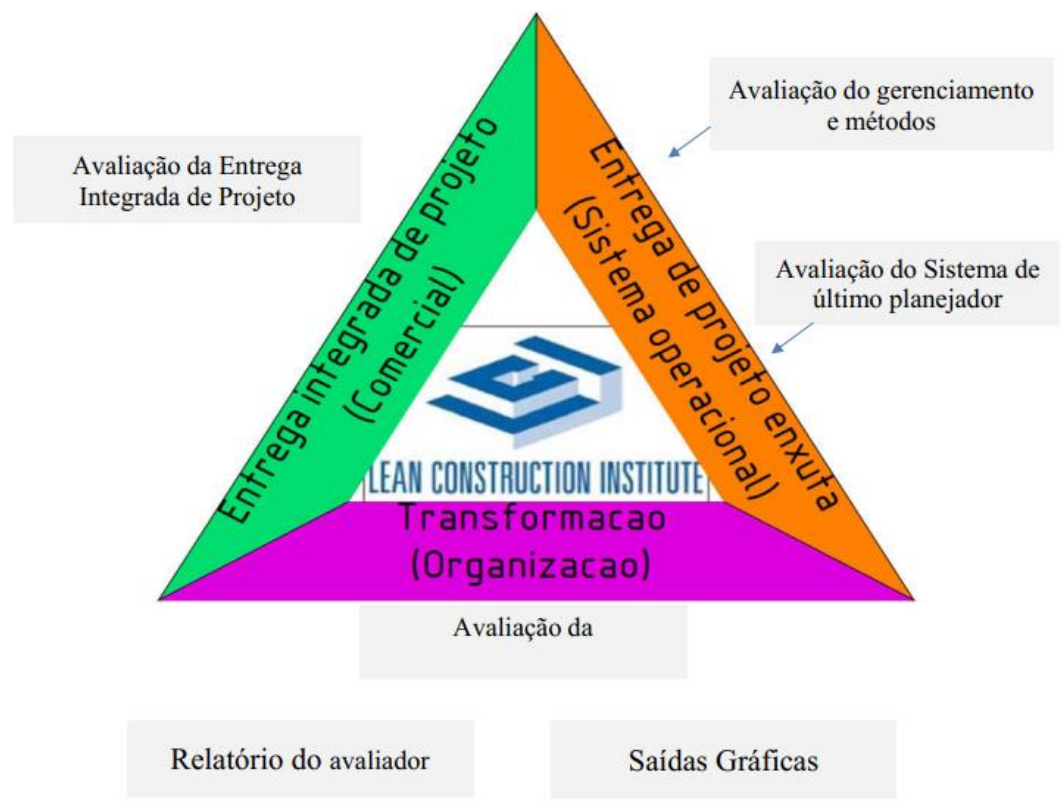

Fonte: Adaptado de Lean Construction Institute (2016). 
A empresa responsável pelo empreendimento estudado não utiliza formalmente CE, apesar dos profissionais desta conhecerem a filosofia e os pesquisadores terem identificado práticas aplicadas despropositadamente. Além disso, existem limitações neste empreendimento que dificultam o pleno desempenho de determinadas práticas da filosofia Lean, como a natureza padronizada das moradias e as exigências do programa público de fomento residencial.

\section{RESULTADOS E DISCUSSÃO}

Após a aplicação do MM, os resultados obtidos são demonstrados a seguir na Figura 3 que apresenta a maturidade da empresa segundo os quatro pontos iniciais de avaliação e características verificadas, sendo duas dessas representações da mesma cor, pois pertencem ao mesmo ponto inicial. A Figura 4 apresenta o gráfico-resumo da avaliação do empreendimento em análise, e por meio desse gráfico pode-se definir o percentual de adesão às práticas da $\mathrm{CE}$, que é de $36 \%$ estando no nível "aprendendo".

Figura 3 - Avaliação segundo a ferramenta Lean IPD

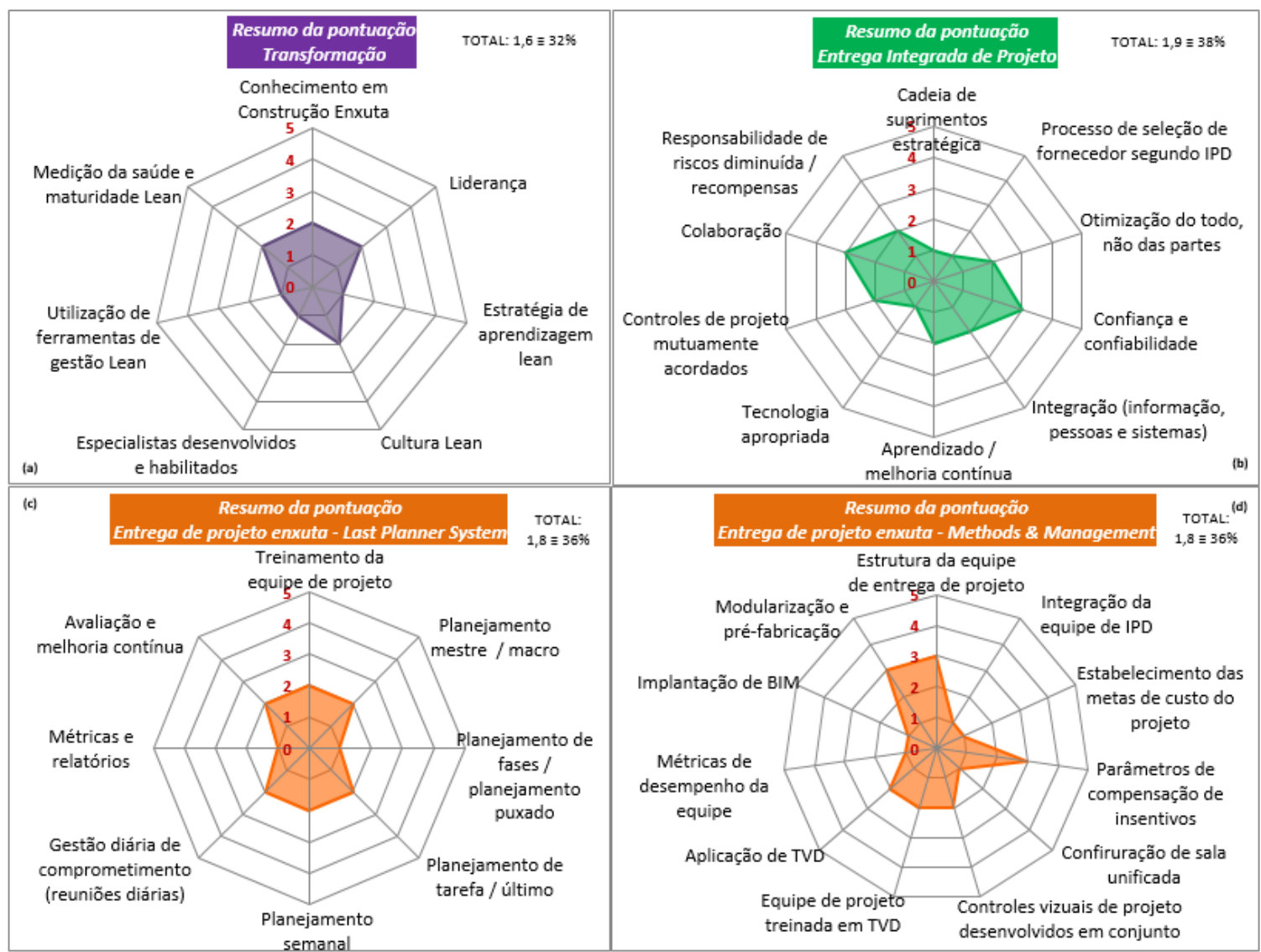

Fonte: Adaptado de Lean Construction Institute (2016).

O gráfico radar (a) da Figura 3 representa a adesão do empreendimento aos itens de avaliação referentes a Transformação na esfera organizacional. Nesta seção a ferramenta avalia oito características da transformação organizacional, observa-se que para quatro destas o empreendimento foi avaliado no segundo nível da escala e as demais no primeiro nível. A média pontual é de 1,6, esse valor corresponde a $32 \%$ de adesão aos atributos apresentados estando dentro do nível de classificação dito como 
"atento", e pode ser verificada na Figura 4, quadrante correspondente à Transformação. Essa avaliação corresponde ao segundo nível da escala de resultado, mostrando que o empreendimento possui baixa implementação de práticas correlacionadas a transformação organizacional em direção ao Pensamento Lean.

\section{Figura 4 - Avaliação segundo a ferramenta Lean IPD, gráfico-resumo}

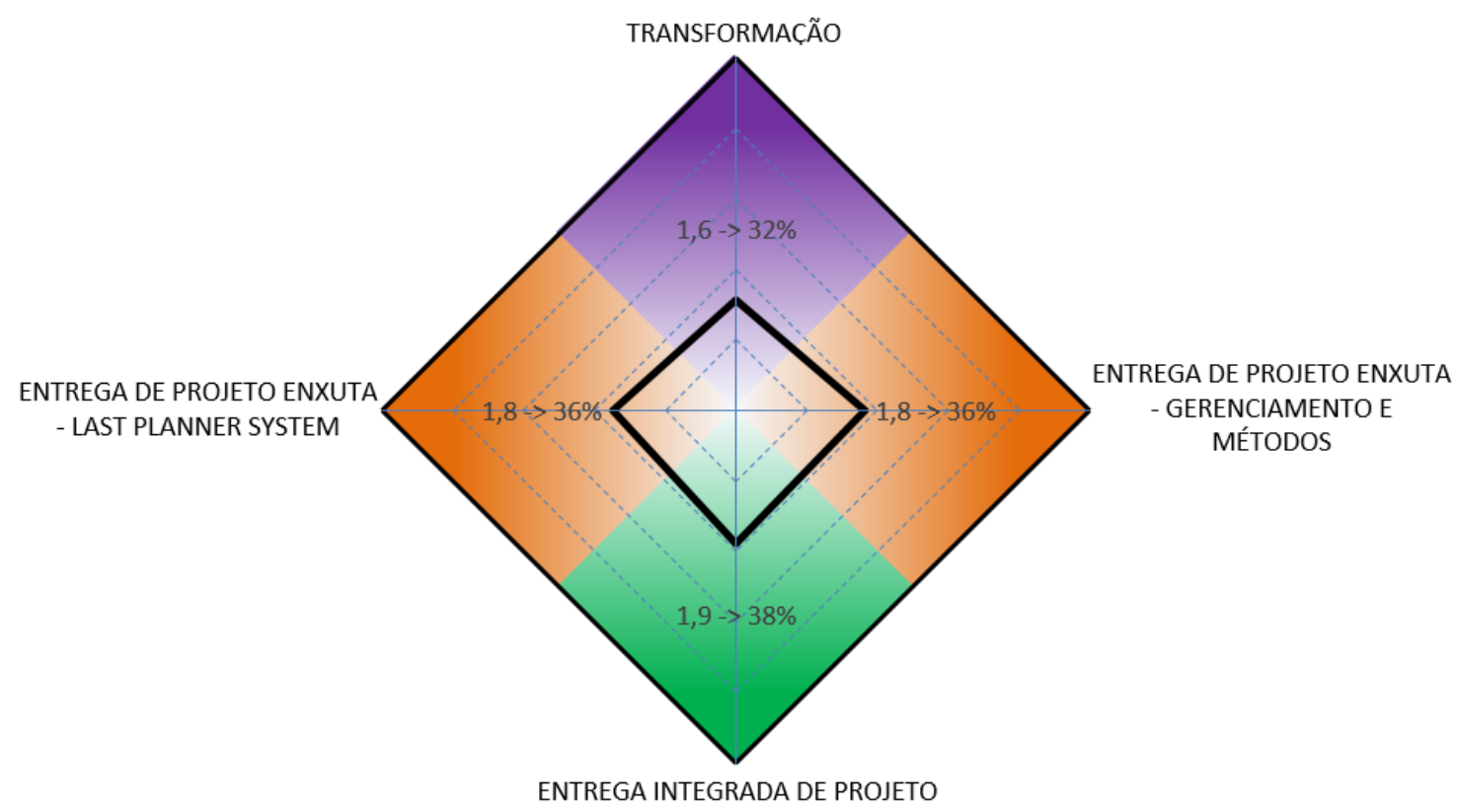

Fonte: Adaptado de Lean Construction Institute (2016).

O gráfico radar (b) da Figura 3 representa a avaliação de dez características referentes a "Entrega Integrada de Projeto (IPD)" em que a avaliação varia de um a três pontos, obtendo média 1,9, conforme observado no quadrante inferior do gráfico-resumo da Figura 4, correspondendo a 36\% de adesão. A avaliação classifica o empreendimento no nível "aprendendo", sendo esse o terceiro nível da escala dessa ferramenta, demonstrando que o projeto aplica práticas relacionadas a IPD, mesmo que de forma não intencional.

Os gráficos (c) e (d) da Figura 3 possuem mesma cor e são relativos a "Entrega de Projeto Enxuto", sendo o (c) focado no Sistema de Planejamento Último (LPS) e o (d) no Gerenciamento e Métodos aplicados. Para o primeiro, o empreendimento pontuou 1,8 na média entre as oito características avaliadas, como verifica-se na área esquerda do gráfico da Figura 4, equivalendo a $36 \%$ de adesão. Para o segundo, a pontuação média para as onze características avaliadas, foi de 1,9 conforme observado na área esquerda do gráfico da Figura 4, sendo equivalente a 38\% de adesão. Para os atributos avaliados relacionados a IPD, o empreendimento obteve classificação dentro do nível "aprendendo", mostrando que o mesmo aplica práticas avaliadas nesses dois questionários apresentados pelo MM.

Realizando a média dos valores obtidos por meio da Figura 4, identifica-se que o empreendimento possui adesão de $36 \%$ aos itens de avaliação representados nos 191 atributos apresentados nessa ferramenta, estando no nível "aprendendo" da escala classificatória desta, assim como para três das quatro áreas que o MM é subdividido, 
SIBRAGEC - ELAGEC 2019 - de 23 a 25 de Outubro - LONDRINA - PR

reafirmando que, mesmo despropositadamente, o projeto utiliza e se beneficia da aplicação de práticas da CE.

\section{CONCLUSÃO}

Por meio desse estudo de caso, foi possível verificar que o MM "Lean IPD” é aplicável a empreendimento de pequeno-médio porte brasileiros já que:

- É coerente a classificação do empreendimento abaixo do nível médio da escala do método sendo que ele não usa formalmente $\mathrm{CE}$ mas foram verificadas utilizações despropositadas dessa filosofia;

- O MM utilizado é difundido internacionalmente e possui ampla abrangência das práticas da CE representados nos 191 atributos avaliados, direcionando o aperfeiçoamento da filosofia ao longo da jornada;

- Mais de $90 \%$ da ICC brasileira é composta por empresas de pequeno-médio porte, conforme dado do Serviço Brasileiro de Apoio às Micro e Pequenas Empresas (SEBRAE, 2018) e Câmara Brasileira da Indústria da Construção (CBIC, 2017);

- Segundo Carvalho e Scheer (2017), é baixa a utilização da CE nas empresas construtoras brasileiras.

Como limitação dessa pesquisa ressalta-se a aplicação em apenas um empreendimento. Para que este MM seja considerado amplamente adaptado à avaliação da ICC brasileira, e como proposta de trabalhos futuros, faz-se necessária sua aplicação em empreendimentos diversos, inclusive de grande porte. Além disso, é possível fazer a mensuração em relação à totalidade da empresa e não apenas restrita a um empreendimento. Entretanto, a pesquisa exploratória cumpriu o objetivo que foi demonstrar a viabilidade da utilização desta ferramenta para a construção civil brasileira.

\section{REFERÊNCIAS}

BÖES, J. S.; ROCHA, P. H.; CÂNDIDO, L. F.; BARROS NETO, J. D. P. Levantamento de melhores práticas de construtoras cearenses. Em: Encontro Nacional de Tecnologia do Ambiente Construído (ENTAC). Anais... Foz do Iguaçú - PR - BR. 2018

CÂMARA BRASILEIRA DA INDÚSTRIA DA CONSTRUÇÃO - CBIC. Tabela anual com o número de estabelecimentos na Construção Civil por tamanho no Brasil e Grandes

Regiões, 2017. Disponível em: <http://www.cbicdados.com.br/menu/empresas-deconstrucao/estabelecimentos-na-construcao> Acesso em mar. 2019.

CARVALHO, B. S. DE; SCHEER, S. Analysis and Assessment for Lean Construction Adoption: The DOLC Tool. Em: $25^{\circ}$ Annual Conference of the International Group of Lean Construction (IGLC). Proceedings... Heraklion, Grécia, 2017. Disponível em: <http://iglc.net/Papers/Details/1449> Acesso em mar. 2019.

FRANCISCO, H. A.; SERRA, S. M. B.; LORENZON, I. A. Diagnóstico de aplicação da Lean Construction em construtoras das cidades de São Carlos e São Paulo - SP. Em: XIV Em: Encontro Nacional de Tecnologia do Ambiente Construído (ENTAC). Anais... Juiz de Fora MG - BR. 2012.

GOMES, M. M. B.; CAVALCANTE FILHO, J. U. D. P.; BARROS NETO, J. D. P. Planejamento estratégico na indústia da construção civil: áreas, temas, técnicas e métodos. Em: 
SIBRAGEC - ELAGEC 2019 - de 23 a 25 de Outubro - LONDRINA - PR

Encontro Nacional de Tecnologia do Ambiente Construído (ENTAC). Anais... Foz do Iguaçú PR - BR. 2018.

GONTIJO, D. S. M.; SANTANA, J. D. C.; PRADO, A. A. Do. Análise dos requisitos para a implementação da filosofia lean green construction em edificações de pequno porte. Em: Encontro Nacional de Tecnologia do Ambiente Construído (ENTAC). Anais... Foz do Iguaçú PR - BR. 2018.

KOSKELA, L. Application of the new production philosophy to construction. Technical Report. Finland VTT Building Technology. Finland, 1992.

LEAN CONSTRUCTION INSTITUTE. LCI Lean IPD Health and Maturity Assessment Tool 1.0 Arlington - TX - USA, 2016. Disponível em:

<https://www.leanconstruction.org/learning/tools-and-technologies/> Acesso em mar. 2019.

LORENZON, I. A. A medição de desempenho na construção enxuta: estudos de caso. Tese (Doutorado em Engenharia de Produção) - Universidade Federal de São Carlos - UFSCar, 2008.

SAINATH, Y.; VARGHESE, K.; RAGHAVAN, N. Framework for progressive evaluation of Lean Construction Maturity using multi-dimensional matrix. Em: $26^{\circ}$ Annual Conference of the International Group for Lean Construction (IGLC). Proceedings... Chennai, India. 2018. Disponível em: <http://iglc.net/Papers/Details/1561> Acesso em mar. 2019

SALVATIERRA, J. L.; Alarcón, L. F.; López, A.; Velásquez, X. Lean diagnosis for chilean construction industry: Towards more sustainable Lean practices and tools. Em: $23^{\circ}$ Annual Conference of the International Group for Lean Construction (IGLC). Proceedings... Perth, Australia. 2015. Disponível em: <https://bit.ly/2IwDRUX> Acesso em mar. 2019.

SERVIÇO BRASILEIRO DE APOIO ÀS MICRO E PEQUENAS EMPRESAS - SEBRAE. Critérios de classificação de empresas: MEI - ME - EPP, 2018. Disponível em:

<http://www.sebrae-sc.com.br/leis/default.asp?vcdtexto=4154> Acesso em mar. 2019.

SOUSA, D. S. V. De; CÂNDIDO, L. F.; BARROS NETO, J. de P. Medição de desempenho na construção civil: um estudo exploratório com construtoras cearenses. Ambiente Construído, v. 18, n. 1, p. 9-29, 2017.

TEZEL, A.; KOSKELA, L.; AZIZ, Z. Current condition and future directions for lean construction in highways projects: A small and medium-sized enterprises (SMEs) perspective.

International Journal of Project Management, v. 36, n. 2, p. 267-286, 2017.

WOMACK, J. P.; JONES, D. Lean Thinking - banish waste and create wealth in your corporation. Journal of the Operational Research Society, v. 48, n. 11, p. 1144-1150, 1997.

YU, I.; KIM, K.; JUNG, Y.; CHIN, S. Comparable performance measurement system for construction companies. Journal of Management in Engineering, v. 23, n. 3, p. 131-140, 2007.

ZHOU, B. Lean principles, practices, and impacts: a study on small and medium-sized enterprises (SMEs). Annals of Operations Research, v. 241, n. 1-2, p. 457-474, 2016.

\section{AGRADECIMENTOS}

Agradecemos o apoio da Coordenação de Aperfeiçoamento de Pessoal de Nível Superior (CAPES) e da Fundação de Amparo à Pesquisa do Estado de São Paulo (FAPESP), assim como à empresa colaboradora com a pesquisa. 